\title{
Didactiques et disciplines : une relation structurelle
}

Didactics and scholastic discipline : a structural relationship

\section{Yves Reuter}

\section{OpenEdition}

\section{Journals}

Édition électronique

URL : https://journals.openedition.org/educationdidactique/1877

DOI : 10.4000/educationdidactique. 1877

ISSN : 2111-4838

\section{Éditeur}

Presses universitaires de Rennes

\section{Édition imprimée}

Date de publication : 30 juillet 2014

Pagination : 53-64

ISSN : 1956-3485

\section{Référence électronique}

Yves Reuter, «Didactiques et disciplines : une relation structurelle», Éducation et didactique [En ligne],

8-1 | 2014, mis en ligne le 15 septembre 2016, consulté le 25 avril 2022. URL : http:// journals.openedition.org/educationdidactique/1877 ; DOI : https://doi.org/10.4000/ educationdidactique. 1877 


\section{DIDACTIQUES ET DISCIPLINES :}

\section{UNE RELATION STRUCTURELLE}

Yves Reuter

Université Lille 3, Laboratoire Théodile-CIREL (E.A. 4354) 
Je souhaite, en premier lieu, remercier les responsables de l'Association pour des Recherches Comparatistes en Didactique ainsi que ceux de la revue Éducation et Didactique pour avoir pris l'initiative d'organiser le débat autour des conceptions à l'œuvre dans le champ des didactiques quant aux relations entre didactiques et disciplines et quant à la diversité ou à l'unité des didactiques ${ }^{1}$. Ces remerciements sont d'ailleurs d'autant plus sincères, qu'aussi bien le nom de l'association que celui de la revue mettent au singulier le terme "didactique ", ce qui n'est pas sans renvoyer à des positions, implicites ou explicites, voire à des appartenances disciplinaires.

Cette initiative témoigne en tout cas d'une volonté de débattre et, je l'espère, de la reconnaissance que différentes positions sont possibles et légitimes. Et je pense, qu'au-delà d'une expérience en partie problématique dans la revue Éducation et Didactique (Reuter, Cohen-Azria, Daunay, Delcambre et Lahanier-Reuter, 2011), cette journée a pu montrer que les débats peuvent être sérieux sans être agressifs, voire violents.

Je tenterai donc d'expliquer dans cette contribution pour quelles raisons je préfère - nous préférons ${ }^{2}$ au sein de Théodile - employer le terme " didactiques " au pluriel, en relation avec les disciplines scolaires et la manière dont nous interrogeons la notion de discipline. Je poursuivrai en exposant quelles sont nos interrogations face aux multiples désignations émergentes qui ont en commun le fait d'adjoindre une expansion adjectivale au terme de «didactique » et en quoi l'expression «didactiques disciplinaires » me pose problème. J'essaierai encore, chemin faisant, de ne pas esquiver certains problèmes auxquels nous nous confrontons et qui me paraissent loin d'être simples à résoudre.

\section{LES RAISONS D’UN PLURIEL}

J'avancerai ici trois types de raisons qui sont susceptibles d'expliquer et de justifier le pluriel en question : raisons d'ordre épistémologique, raisons d'ordre historique et raisons d'ordre institutionnel.

\section{Raisons d'ordre épistémologique}

En premier lieu, je rappellerai que les didactiques se sont constituées sur la base d'une focalisation sur les contenus, en tant que ceux-ci sont objets d'enseignement et d'apprentissages. Cela a amené Jean-Louis Martinand à poser - au travers d'une expression demeurée célèbre dans nos champs de recherche la responsabilité des didactiques quant aux contenus:

Il n'est pas possible de parler de didactique sans l'exercice de ce qu'on peut appeler « une responsabilité par rapport au contenu » de la discipline. (Martinand, 1987, p. 24)

Cette première proposition nécessite cependant quelques éléments de clarification. En premier lieu, j'emploie à dessein le terme de "contenus", dans la mesure où il me parait difficile de parler d'objets d'enseignement et/ou d'apprentissages sans reconnaitre leur diversité (Daunay et Reuter, 2012 ; Reuter, 2010) : savoirs, savoir-faire, rapports à, valeurs, manières de penser, de discourir, d'agir ${ }^{3} \ldots$

En second lieu, je ne vois pas comment on pourrait analyser les contenus sans prendre en compte leur modalités organisationnelles et notamment la modalité disciplinaire prégnante à l'école, envisagée comme forme scolaire (Thevenaz - Christen, 2008 ; Vincent, 1980 ; Vincent, dir. 1994 ; Vincent, 2012). C'est d'ailleurs cette modalité disciplinaire qui les fait exister en tant que contenus d'enseignement et d'apprentissages dans la mesure où - et sur ce point je suis tout à fait en accord avec Chervel (1988, p. 90) - la visée fondamentale des disciplines est de «fabriquer de l'« enseignable » ». Cela étant posé, cela ne signifie pas que les didacticiens n'étudient, ou ne devraient étudier, que des formes disciplinaires «établies». Cela signifie qu'à l'école les modalités organisationnelles des contenus, quelle que soit leur diversité, se constituent en relation, voire en référence, aux disciplines et au système auquel elles appartiennent/ qu'elles constituent. Si j'insiste ainsi sur l'école, c'est parce que cette entrée par les contenus s'est historiquement effectuée en se centrant sur celle-ci, ce qui ne signifie d'ailleurs pas que les études didactiques se cantonnent à l'école ou devraient le faire mais que, lorsqu'elles en « sortent », elles le font en référence au terrain scolaire : soit par comparaison entre institutions, soit pour mieux comprendre les relations entre scolaire et extrascolaire ${ }^{4}$. C'est, en quelque sorte, leur marque de fabrique, leur spécificité par rapport aux autres disciplines de recherche qui s'intéressent à ces questions. 
Cela indique complémentairement que, loin d'être des idéologues qui seraient susceptibles de discourir sur tout, les didacticiens ont besoin de circonscrire un domaine du monde. En effet, fondamentalement, ce qui est en jeu à mon sens dans le geste inaugural qui a amené à constituer un nouveau champ disciplinaire, c'est le refus du " généralisme » qui est à l'œuvre dans nombre d'autres disciplines qui s'intéressent aux phénomènes éducatifs : le didacticien n'est pas le spécialiste de tous les contenus, en tous lieux sociaux; il ne peut l'être, s'il veut posséder une certaine maitrise de ceux qu'il tente d'appréhender.

\section{Fonctionnements institutionnels}

Les fonctionnements institutionnels renvoient ici aux modes de formation, d'insertion et de positionnement des didacticiens. De fait, les didacticiens, du moins ceux des premières générations, ont été formés dans un cadre disciplinaire, souvent à un haut niveau académique, ce qui pose d'ailleurs le problème des modalités de leur reproduction ${ }^{5}$. Ils sont inscrits - au moins en France - dans une double appartenance virtuelle dans les sections du CNU : celle de leur discipline universitaire d'origine et celle des Sciences de l'éducation, section qui a su à maintes reprises compenser leur exclusion de l'espace de légitimité de leur discipline d'origine. Ils continuent à suivre les travaux de recherche dans leurs champs de référence afin de se tenir au courant des évolutions disciplinaires, des débats théoriques, etc. Ils peuvent aussi continuer à participer à la vie scientifique de ces champs (recherches, jurys de concours ou de thèses, communications dans des journées d'étude et des colloques, articles dans des revues...). Leur positionnement - catégorie qui renvoie en l'occurrence à leurs sphères d'intervention et aux acteurs avec lesquels ils ont des contacts privilégiés - est donc à l'intersection entre le champ de l'éducation et ceux plus spécialisés de leur discipline d'origine et de la discipline scolaire dont ils sont les spécialistes.

\section{Le développement historique}

Ce que j'appelle développement historique renvoie aux modes d'organisation des didactiques. De fait, ceux-ci s'avèrent différents selon les didactiques: Instituts (tels les IREM pour les mathématiques); associations plus ou moins importantes (que ce soit en nombre ou en quantité d'adhérents) et mode de fonctionnement de celles-ci ; communautés plus ou moins conviviales; universités d'été ; revues en plus ou moins grand nombre...

Le développement historique réfère aussi aux thématiques ou problématiques plus ou moins développées selon les champs didactiques, aux concepts privilégiés ou encore aux relations avec les disciplines possiblement contributoires. Nous avons ainsi avancé (Daunay et Reuter, 2012 ) le fait, que le concept de transposition didactique (Chevallard, 1985/1991), issu de la sociologie (Verret, 1975), ait connu une telle postérité en didactique des mathématiques ou que la question du curriculum soit ainsi thématisée en didactique de la technologie, avait sans doute à voir avec les disciplines scolaires, leur histoire, les conceptions qu'en ont leurs acteurs...

Le développement historique renvoie encore aux manières spécifiques qu'ont chacune des didactiques de traiter des questions auxquelles elles sont confrontées. Par exemple, sur la question de l'erreur, sur laquelle je travaille depuis maintenant près de trente ans, on voit bien que les didactiques se différencient selon la manière dont elles posent ou non la question du statut de l'erreur, selon l'importance qu'elles lui accordent, selon les concepts (interlangue, représentations...) avec lesquels elles la mettent en relation (Reuter, 2013).

Les didactiques sont donc différenciées et dans un rapport qui me parait indéniable à des disciplines scolaires. Les didacticiens sont des spécialistes de telle ou telle discipline scolaire et des disciplines référentes ou d'une partie d'entre elles. Cela implique que ce rapport est particulièrement complexe, dans la mesure où, par exemple, il n'existe pas de relation univoque entre discipline universitaire et / ou de recherche et discipline scolaire. Cela nous a amenés à parler de constellation disciplinaire (Reuter et Lahanier-Reuter, 2004/2007) pour désigner les rapports que les didacticiens construisent à ce qui est de l'ordre des disciplines scolaires d'un côté et à ce qui est de l'ordre de disciplines référentes (ou potentiellement référentes) dans le champ universitaire et /ou dans le champ de recherche, de l'autre ${ }^{6}$.

Il me semble, en tout cas, qu'à partir des éléments présentés ici, essentiellement les relations des didactiques aux contenus, à leurs modalités organisationnelles et aux disciplines, on peut sans doute mieux comprendre le pluriel que nous défendons. On peut 
sans doute aussi mieux comprendre en quoi l'expression « didactiques disciplinaires »a, pour nous, un parfum quelque peu pléonastique.

\section{LE CONCEPT DE DISCIPLINE}

Cette relation que nous estimons fondamentale aux disciplines nous a en tout cas conduits à effectuer un travail critique sur la notion de discipline (Reuter et Lahanier-Reuter, 2004/2007) qui, plusieurs années après l'article de référence d'André Chervel (1988), n'a pas véritablement fait l'objet d'une réflexion à la hauteur de son importance pour les didactiques. C'est donc dans cette perspective que je souhaite rappeler, trop brièvement sans doute, les recherches que nous avons menées, en insistant sur quelques déplacements théoriques plus récents ${ }^{7}$. Il me semble en effet que les travaux, entrepris depuis plusieurs années déjà, ont permis de soumettre au débat quelques propositions, qui portent sur la définition même des disciplines, sur les manières de les décrire, sur leur « statut » ainsi que sur leur mode d'existence et leurs variations.

\section{Définition}

La définition que j'ai proposée dans le Dictionnaire des concepts fondamentaux des didactiques (Reuter, dir., 2007/2010, p. 85), et que je maintiens encore, est la suivante :

Une discipline scolaire est une construction sociale organisant un ensemble de contenus, de dispositifs, de pratiques, d'outils... articulés à des finalités éducatives, en vue de leur enseignement et de leur apprentissage à l'école.

Tous les éléments de cette définition de travail sont nécessairement à discuter, ainsi qu'il se doit dans un espace de recherche. Trois d'entre eux me paraissent en tout cas fondamentaux :

-parler de discipline scolaire implique de distinguer soigneusement entre " disciplines de recherche ", en mouvement et constamment en cours de (ré)élaboration entre chercheurs, "disciplines universitaires », enseignées par des spécialistes plus ou moins «pointus », fournissant une vision de haut niveau mais élargie et plus stable à de possibles futurs spécialistes $^{8}$ et enfin « disciplines scolaires » qui sont des constructions spécifiques destinées à un public plus jeune, dispensées par des enseignants qui n'en sont pas forcément des spécialistes, sans vocation aucune à former des spécialistes et référant souvent à des disciplines universitaires fort différentes ;

-parler de construction sociale insiste sur deux dimensions complémentaires : d'une part, sur le fait qu'une discipline scolaire n'a rien de naturel, il s'agit d'une construction sociale, institutionnelle, d'autre part sur le fait qu'elle est constamment en débat et en renégociation au sein de l'école et de la société, même si les modalités et l'intensité de ces débats sont très variables selon les disciplines;

-parler d'organisation porte l'accent sur le fait qu'une discipline articule des composantes de nature différente qu'il convient d'analyser non seulement pour elles-mêmes mais aussi au regard de l'homogénéité ou de l'hétérogénéité de l'ensemble auquel elles participent.

\section{Décrire la discipline}

Il me semble aussi que la proposition d'axes descriptifs n'est pas négligeable, dans la mesure où un tel outil n'était pas véritablement disponible sur la scène théorico-méthodologique. Je rappellerai donc ici les principaux axes que nous avons proposés :

-des composantes structurelles : catégories de contenus, modes d'organisation de ces contenus, exercices, modalités de contrôle, modalités de travail, formes de mise en œuvre de matérielle (espace, temps, tenue, outils...)...;

-des visées, en distinguant ici les visées proprement disciplinaires, celles qui sont communes aux différentes disciplines et propres à l'école et celles qui excèdent le cadre scolaire : en d'autres termes, les disciplines visent à construire un sujet disciplinaire, un sujet scolaire et un sujet extrascolaire (Reuter, 2005b);

-des fonctionnements institutionnels : désignations des disciplines (et changements ou luttes autour de ces désignations), modes de présence (temps alloué, horaires, poids dans les évaluations et dans l'orientation...), modes de formation des acteurs...;

-des relations aux espaces "externes " à l'école : aux espaces théoriques, aux champs de pratiques sociales, à des institutions spécifiques ${ }^{9}$, aux pratiques extrascolaires des élèves, à la société en tant qu'es- 
pace de débats (ce que j'ai appelé la plus ou moins grande « sensibilité sociale » d'une discipline ${ }^{10} \ldots$ );

-des variations diachroniques et synchroniques, sur lesquelles je reviendrai immédiatement après.

Il est clair pour moi, une fois encore, que toutes les catégories proposées sont discutables. Il est clair aussi que demeurent plusieurs problèmes cruciaux que j'ai mentionnés à diverses reprises. Le premier est celui des frontières initiale et finale des disciplines. À quels moments du cursus et selon quels critères, peut-on dire qu'une discipline commence ou se termine? Le deuxième problème renvoie une difficulté méthodologique réelle. Il est, en effet, impossible d'étudier une discipline dans toute son amplitude et dans toutes ses variations. Dès lors, à quel moment et sur quelles bases, décide-t-on que la description réalisée est satisfaisante ? Le troisième problème est celui du faire disciplinaire (Reuter, 2005a). Il porte sur la pratique de la discipline. Quand «fait-on de la discipline» (Français, Mathématiques, Éducation Physique et Sportive...), c'est-à-dire quand peut-on dire que les élèves font telle ou telle discipline ? Et quand peut-on dire qu'ils n'en font pas/plus, que ce soit dans l'espace de la classe ou en dehors de cet espace ? Par exemple, on peut être en EPS sans en faire (déplacement, changement de tenue...). Par exemple encore, si on peut s'accorder sur le fait qu'un élève qui écrit une lettre d'amour en cours de mathématiques ne fait pas des mathématiques, qu'en est-il d'un élève qui recopie sur son voisin lors d'un contrôle de mathématiques ? Par exemple aussi, peut-on parler d'un faire disciplinaire lorsqu'un élève lit chez lui, sur les conseils de son enseignant, pour améliorer son orthographe ou « enrichir» son vocabulaire ? Par exemple encore, qu'en est-il d'un élève qui construit comme une situation mathématique une situation imposée à l'entrée du collège où il ne peut indiquer qu'un seul nom d'ami avec lequel il souhaiterait être en classe : il étudie ainsi ce que ses deux amis et lui doivent effectuer comme demandes afin qu'ils puissent tous trois se retrouver dans la même classe. Ici encore, je ne dispose pas de véritable réponse mais ce questionnement sur le faire disciplinaire, me paraît tout aussi important que sous-estimé. La dernière question, que j'ai aussi formulée à plusieurs reprises (notamment, Reuter 2011, p. 50-51) porte sur la " disciplinarité » des contenus. Qu'est-ce qui fait qu'un contenu peut être considéré comme disciplinaire, c'est-à-dire d'une part non « commun » et, d'autre part, ne relevant pas de telle ou telle autre discipline ? Cela me parait loin d'être évident et la proposition, au demeurant très stimulante, de Jean-Pierre Astolfi (2010, p. 217), ne simplifie pas réellement, à mon sens, les choses :

Aucune discipline n'a le monopole d'un contenu d'enseignement (puisqu'on n'apprend pas à lire qu'en français, ni à calculer qu'en mathématiques), mais chaque contenu a besoin d'une discipline de référence qui prenne en charge sa structuration.

\section{Le statut disciplinaire}

Complémentairement, nous avons aussi tenté de réfléchir à la difficile question de ce que j'appellerai ici, faute de mieux, le «statut disciplinaire ».

Cela concerne d'une part, le fonctionnement, l'histoire, les visées, l'autonomie... qui renvoient à la question de la distinction entre disciplines et « éducations à », voire quasi-disciplines. Cela renvoie, d'autre part, à la question des dispositifs ${ }^{11}$, souvent conçus initialement comme des moyens de lutter contre le «cloisonnement disciplinaire » mais qui finissent souvent, eux-aussi ${ }^{12}$, par fonctionner comme des disciplines scolaires (ou des quasi-disciplines).

Cela renvoie enfin au travail considérable de Chervel $(1988,1998,2006)$ dans le domaine de l'histoire des disciplines et à la distinction possible entre, d'une part, matière scolaire et d'autre part, disciplines comme composantes, voire comme entités singulières, caractérisées par une autonomie de contenus, d'exercices, de modalités d'évaluation, voire une histoire spécifique ${ }^{13}$. Je dois dire que, sur cette question, j'hésite encore dans le maniement de ces catégories, dans la mesure où, par exemple, il ne me semble pas que les composantes « histoire » et " géographie » de la matière " histoire-géographie » ${ }^{14}$ soient du même ordre que les composantes " orthographe ", "grammaire", « conjugaison », « vocabulaire », « lecture »... de la matière "Français ». Dans la mesure aussi où les composantes peuvent s'emboiter sur plusieurs niveaux et sont susceptibles d'être combinées selon des modalités variées. C'est pour cela que, tout en tenant cette question pour essentielle, je m'en tiens, au moins provisoirement, à une alternance terminologique ${ }^{15}$ qui évite de trancher entre " matière » et « discipline » pour le niveau « global » et j'emploie les désignations de composantes ou de sous-disciplines pour les autres niveaux. 


\section{Configurations disciplinaires et « noyau dur»}

Il me semble que nous avons sensiblement plus avancé sur la question des modes d'existence ou, dit sous une autre forme, sur la question des variations et des modes d'actualisation différents des disciplines selon les pays, les époques, les moments du cursus, les filières, les modes de travail pédagogique (Lesne, 1977) et les espaces (Reuter et Lahanier- Reuter 2004/2007), ce que nous avons appelé des configurations disciplinaires. Nous avons notamment distingué, et c'est fondamental pour nous, des espaces différents d'actualisation des disciplines : un espace de prescriptions (par exemple, en France, les textes « officiels ${ }^{16}$ ), un espace de recommandations (formation, inspection, associations, manuels...), un espace de pratiques et un espace de reconstruction par les acteurs $^{17}$, ce qui permet, par voie de conséquence, de différencier des modes d'actualisation différents des disciplines : des configurations disciplinaires prescrites, recommandées, pratiquées, (re)construites par les acteurs. Portant l'accent sur des modes d'existence différents, notre approche est donc à l'opposé d'une " essentialisation» des disciplines.

Ces configurations sont cependant coréférentes et, conséquemment, la question du "noyau dur » est, pour moi, complémentaire de celle des configurations disciplinaires mais finalement tout aussi peu pensée. Il s'agit ici d'essayer de définir ce qui caractériserait « centralement» une discipline, en tant que catégorie scolaire et sociétale, relativement partagée, de moyenne voire de longue durée, susceptible de regrouper les différentes configurations disciplinaires qui lui sont attribuées, à elle et non à une autre discipline et, solidairement, d'éclairer l'espace des conflits qui concernent, voire qui constituent, telle ou telle discipline.

Dans cette perspective, j'ai proposé de construire ce «noyau dur » autour de trois éléments principaux (Reuter, 2011):

-un domaine et des objets du monde privilégiés : en ce sens, toute discipline est un découpage du monde ;

-une perspective sur ce découpage du monde (par exemple, la discipline Français se caractériserait, entre autres, par une valorisation et une appréhension formelle du langagier et du discursif) : en ce sens, une perspective construit donc, en articulation avec le découpage, un rapport au monde ; -des tensions structurantes qui déterminent, en grande partie, les configurations disciplinaires possibles (par exemple, en Français, entre unification et différenciation langagière, entre description des faits textuels et prescription, entre acquisition de normes et imposition de surnormes, entre autonomie et ancillarité, entre investissement et distance ${ }^{18}$.

\section{Modes d'appropriation des disciplines}

Cela nous a encore permis, et ce n'est pas là le moindre intérêt de cette entrée, de développer des travaux, que nous estimons importants pour la compréhension de nombre de problèmes, autour du concept de conscience disciplinaire (Reuter, 2003, 2007 ; Cohen-Azria, Lahnier-Reuter et Reuter, dir., 2013), entendu comme « la manière dont les acteurs sociaux, et en premier lieu, les sujets didactiques - élèves mais aussi enseignants - reconstruisent telle ou telle discipline. » (Reuter, dir., 2007/2010, p. 41). On peut appréhender, grâce à cet éclairage, comment les « mêmes » matières sont reconstruites différemment selon les élèves, comment certaines sont " claires * pour la majorité d'entre eux et d'autres non, ou encore certaines caractéristiques de chacune d'entre elles (par exemple l'homogénéité des Mathématiques opposée à l'hétérogénéité du Français ou de l'EPS, ou encore la dimension normative du Français), ce qui n'est pas sans contribuer à engendrer des différences de performances, des malentendus, etc.

Cela s'articule à un déplacement important, peut-être le plus important que nous ayons effectué ces derniers temps, qui consiste à étudier les disciplines en tant qu'espaces vécus, c'est-à-dire comme des espaces qui sont des lieux et des sources de vie et d'émotions. Cela nous amène à analyser les effets que produisent les fonctionnements disciplinaires. Il s'agit, notamment dans une recherche en cours soutenue et financée par la Sauvegarde du Nord ${ }^{19}$, de comprendre les manières dont les élèves existent dans les disciplines, c'est-à-dire leurs différents modes d'être, de ressentir, de se positionner... dans ces espaces d'enseignement et d'apprentissages et, complémentairement, de comprendre ce qu'ils en retirent et ce qui leur en reste (au même titre que les contenus).

Cela signifie que je suis ici en désaccord avec Jean-Pierre Astolfi lorsqu'il écrivait (Astolfi, 1997, p. 99) : 
Yves Reuter

Lorsqu'on demande aux didacticiens comment ils prennent en compte l'affectivité des élèves, ils répondent souvent un peu embarrassés qu'à la vérité ils n'en font rien ! Mais il ne faut pas se méprendre sur la signification d'une telle réponse. Dire qu'on n'en fait rien ne signifie pas qu'on la néglige, encore moins qu'on la méprise. Simplement, la didactique ne va pas redoubler ce que font très bien les autres chercheurs, psychologues, sociologues ou psychanalystes. Ce n'est pas là-dessus que leur travail met l'accent parce qu'ils cherchent, eux, à explorer d'autres voies spécifiques, plus proches du fonctionnement et des situations scolaires.

Ce désaccord tient à deux raisons principales. $\mathrm{La}$ première est, qu'à mon sens, cette dimension du vécu est une composante à part entière « $d u$ fonctionnement et des situations scolaires », trop souvent encore ignorée dans les recherches en didactiques, alors que tous les acteurs interrogés s'accordent sur son importance. La seconde raison consiste en ce que le traitement des autres disciplines de recherche, aussi intéressant soit-il, est insatisfaisant pour les didactiques, justement en ce que ces disciplines ne construisent pas cette question dans une perspective didactique. Nous travaillons donc à préciser les caractéristiques de cette perspective, avec notamment :

- une visée principale qui est de mieux comprendre les caractéristiques des fonctionnements disciplinaires ;

- une focalisation sur les contenus, les disciplines et les configurations disciplinaires plutôt que sur les dimensions scolaires, pédagogiques ou psychologiques ;

- un centrage sur du "générique » (en quoi tels contenus ou dispositifs disciplinaires produisent, de manière récurrente, tels effets sur telles catégories d'élèves) plutôt que sur du « singulier » (ce que cela révèlerait de l'histoire et des problèmes de tel ou tel élève).

J'ajouterai encore que cette entrée peut permettre de penser autrement des questions, telles la violence ou le décrochage scolaires, sur lesquelles je regrette que les didacticiens se fassent peu entendre alors qu'ils auraient, à mon sens, bien des choses à dire et sans doute favoriser de nouvelles modalités de dialogue avec les autres disciplines de recherche qui s'intéressent à l'éducation.

\section{Système disciplinaire}

Il me semble aussi que nous avons mis du temps pour saisir l'importance de la notion de système disciplinaire $^{20}$. Cette notion me parait pourtant particulièrement importante pour deux raisons au moins.

D'une part, parce qu'une discipline scolaire ne se comprend, en tant que construction à la fois relativement commune $e^{21}$ et relativement singulière, que dans ses relations avec les autres disciplines au sein de modalités organisationnelles qui l'intègrent, de systèmes qui varient selon les différents espaces de prescriptions, de recommandations, de pratiques, selon les pays, les époques, selon les modes de travail pédagogique, selon les moments du cursus...

D'autre part, ou plutôt complémentairement, il m'apparaît de plus en plus, notamment dans la recherche sur le vécu disciplinaire que je viens d'évoquer, que la reconstruction du système disciplinaire, très variable selon les sujets scolaires, fait partie intégrante de la conscience disciplinaire, organisant de différentes manières les disciplines en « blocs » ${ }^{22}$, les hiérarchisant, les opposant, isolant l'une ou l'autre comme absolument singulière.

\section{DE QUELQUES REMARQUES SUR QUELQUES CRITIQUES (PAS FORCÉMENT BIEN ATTENTIONNÉES)}

Le retravail de la notion de discipline que je viens d'évoquer, trop brièvement sans doute, est pensé non comme un dogme qu'on répéterait incessamment dans une perspective apologétique mais comme la construction d'un cadre heuristique sans cesse à interroger et à remettre en cause, ce qui explique que je tienne à l'accompagner systématiquement des questions qui se posent ${ }^{23}$. Il me semble qu'il nous prémunit, en tout cas, contre l'accusation de réinvestir "simplement » les découpages et les fonctionnements institutionnels, puisque justement nous travaillons incessamment à les interroger ainsi que je viens de le montrer.

Complémentairement, il me semble que ceux qui avancent cette critique pourraient peut-être s'interroger plus avant sur ce que serait un travail en sciences humaines et sociales qui voudrait totalement s'affranchir des réalités sociales. Comment prétendre à une dimension critique sans prendre en compte les constructions sociales? J'avancerai, par voie de consé- 
quence, que notre vigilance critique est dès lors plus conséquente que celle de ceux qui nous critiquent sans se livrer à un tel travail de déconstruction.

Et j'ajouterai encore que cette réflexion critique est d'autant plus importante à mener que le refoulé des didacticiens, à l'instar de tout autre refoulé, ne cesse de se manifester pour peu qu'on y soit attentif. Ainsi, nombre de ceux qui cherchent à prendre leurs distances avec la notion de didactiques (au pluriel) ou avec les disciplines ont, de fait, beaucoup de difficultés à dissimuler leur origine disciplinaire. Celle-ci se manifeste en effet incessamment, que ce soit dans leur manière d'écrire, par exemple dans le goût que peuvent avoir certains d'entre eux pour les symboles ou les formules mathématiques; que ce soit dans leur assurance quant à penser que les modèles qu'ils construisent seraient valables pour toutes les disciplines; que ce soit dans leurs discours sur les savoirs qui seraient plus « assurés » que ceux d'autres disciplines... Cela les conduit d'ailleurs parfois, bardés de certitudes, à intervenir sur le terrain des autres didactiques, revendiquant ainsi sans peut-être s'en rendre compte, une hiérarchie disciplinaire identique à celle du secondaire (Reuter, 2008).

\section{LA QUESTION DES DÉSIGNATIONS}

J'en arrive maintenant à la question, complémentaire pour moi, de la multiplication des désignations. Je partirai pour traiter cette question d'un constat, appuyé, entre autres, par les analyses très précises de Joël Lebeaume (2012, p. 53-54), celui de la floraison d'expressions désignant les didactiques sous la forme d'un syntagme composé d'un nom auquel est accolé un adjectif (didactique clinique, didactique cognitive, didactique comparée, didactique curriculaire, didactique professionnelle...) ou plus rarement sous forme d'un nom composé (sociodidactique). Cela me parait renvoyer à diverses questions et soulève des problèmes différents.

\section{Des variations désignatives et de leurs possibles raisons}

Cela peut par exemple référer, ainsi que l'indique Joël Lebeaume (2012), à une entrée privilégiée des recherches en didactiques. Ainsi, on pourrait distinguer, à le suivre, une didactique des apprentissages (centrée principalement sur les conditions de l'étude, les contenus et les conditions de leur appropriation), une didactique des curriculums (centrée essentiellement sur la constitution et l'évolution des disciplines) et une didactique des enseignements (centrée prioritairement sur les pratiques des maitres et la formation des enseignants). Il s'agit alors selon moi, au travers de telles désignations, de spécifier les grandes problématiques possibles du champ des didactiques et d'afficher celle à laquelle on se rattache. Demeurent à préciser les modalités et les visées internes et/ou externes de cet affichage.

Cela peut encore renvoyer à un mode d'éclairage particulier, en relation avec des cadres théoriques revendiqués, voire avec des méthodes de recherche empruntées à des disciplines de recherche connexes. Il s'agit alors, ici encore à mon sens, d'une spécification interne des didactiques (voir, par exemple, la sociodidactique ${ }^{24}$ ) mais qui, cette fois-ci, renvoie non seulement à un éventail d'entrées possibles mais encore à des débats entre courants.

Dans d'autres cas (voir par exemple le cas de la psychologie clinique ${ }^{25}$ ), on pourrait se demander, avec Joël Lebeaume, si cela ne mène pas à un renouvellement de l'entrée (psycho) pédagogique, en ce que les études s'en revendiquant ne mettent pas toujours en focale les contenus, mais il me semble qu'on pourrait tout aussi légitimement défendre la position selon laquelle il s'agit, là encore, d'une spécification interne des didactiques ou encore d'un positionnement face à d'autres disciplines dans un domaine particulier (celui des Activités Physiques Sportives et Artistiques).

Dans d'autres cas encore (par exemple, celui de la didactique cognitive ${ }^{26}$ ), il me semble qu'il s'agit plutôt d'un croisement disciplinaire qui conduit à s'interroger pour savoir s'il s'agit encore de didactiques (par exemple d'un courant interne), s'il s'agit de la constitution d'une nouvelle discipline au croisement de disciplines antérieures ou s'il s'agit d'un entre-deux, au statut encore indéterminé.

Le problème de la didactique professionnelle ${ }^{27}$ est pour moi sensiblement différent. Les problèmes posés ne relèvent pas de la qualité des travaux menés dans ce champ qui, au même titre que ceux de bien d'autres disciplines connexes d'ailleurs, intéressent au plus haut point les didacticiens. Ils résident, selon moi, dans la désignation disciplinaire elle-même, dans les fonctionnements adoptés, dans la prétendue nouveauté de cette entrée et, finalement, dans le 
généralisme. En effet, ces travaux s'inscrivent dans le continent de l'analyse du travail et je persiste à ne pas voir le gain de clarification qu'apporte en l'occurrence la désignation de didactique professionnelle. Cela d'autant plus que, sur le terrain de l'enseignement, qui n'est d'ailleurs pas le terrain d'origine de ces travaux, on peut constater des pratiques de généralisation ou de directions de thèses qui font fi de la spécialisation disciplinaire, comme si l'enseignement au même titre que les apprentissages n'étaient pas contraints et spécifiés par les contenus, ce qui me parait pourtant constituer une des hypothèses fondatrices des didactiques. J'ajouterai encore que la revendication de nouveauté de l'entrée par la question de l'enseignement, qui a accompagné l'émergence de ce courant dans le champ des didactiques, me parait relever au mieux d'une ignorance surprenante quant aux recherches menées dans nombre de didactiques, depuis leur origine, sur le travail enseignant (Reuter et Delcambre, 2006).

\section{Didactiques, didactique comparée, didactique générale}

Demeure le cas de la didactique comparée dont le projet est pour moi fondamental pour deux raisons : d'abord, en ce qu'il permet de préciser les spécificités de la discipline dont s'occupe chaque didactique et de penser les spécificités mêmes des fonctionnements de chaque didactique, au travers de la comparaison avec les autres disciplines et les autres didactiques ; ensuite en ce qu'il permet de réfléchir à ce qui constituerait les points communs des didactiques et quel est le statut épistémologique de cette convergence. Cela n'est d'ailleurs pas sans reposer la question de la didactique générale ${ }^{28}$ pourvu, à mon sens, que la distinction entre la perspective de la pédagogie et celle de la didactique générale soit maintenue et pourvu que la dialectique entre didactiques, didactique comparée et didactique générale soit scrupuleusement établie.

Mais demeure complémentairement le fait que ce projet - celui de la didactique comparée - se développe difficilement, à voir le faible nombre de membres de l'Association, et au risque de trois dérives :

-la première consisterait à reproduire sous un autre nom ce que chaque didactique effectue depuis son origine, à savoir des comparaisons internes (entre situations, entre classes, entre moments du cursus...) et, par voie de conséquence, à ne rien apporter de nouveau à l'existant ;

-la deuxième consisterait à vouloir imposer des modèles théoriques, issus de telle ou telle didactique, à l'ensemble des communautés dans l'ignorance de leurs travaux respectifs et des spécificités disciplinaires ;

- la troisième consisterait à vouloir occulter l'origine disciplinaire d'un certain nombre de ses thuriféraires, origine pourtant bien visible (Mercier, Sensevy et Schubauer-Leoni, 2002), développant ainsi un point de vue se voulant surplombant, mais se révélant lacunaire et tributaire de sa discipline et de sa didactique d'origine.

Cette dernière dérive possible signale d'ailleurs l'illusion selon laquelle un unique chercheur pourrait être spécialiste de toutes les disciplines, de tous leurs contenus, de toute l'histoire de toutes les didactiques et interroge la création de postes de didactique comparée, stratégie potentiellement dangereuse à mon sens, en ce qu'elle peut entrainer le dommage irrémédiable de la perte du travail en commun de spécialistes des différentes disciplines, perspective pourtant particulièrement stimulante justement ouverte par le projet comparatiste.

\section{L'expression « didactiques disciplinaires » comme tentative d'imposition}

Il n'en demeure pas moins, pour en revenir à ma question, qu'à un moment historique où les didactiques sont menacées pour de multiples raisons, on se trouve face à une démultiplication des appellations qui brouille le champ de recherches et sa perception. Cette démultiplication est engendrée, classiquement, par la volonté de se démarquer, c'est-à-dire de reprendre les attributs d'une référence tout en s'en différenciant. Cette démultiplication génère conséquemment la nécessité, chez les acteurs qui tentent d'imposer leur marque à ces courants, d'affubler les didactiques originelles dont elles souhaitent se démarquer d'une appellation nouvelle et pléonastique « didactiques disciplinaires ». J'en vois clairement les risques, je n'en perçois pas bien les avantages. 


\section{CONTINUONS LE DÉBAT}

Comme on peut s'en rendre compte, ma contribution engendre finalement plus de questions qu'elle n'apporte de réponses. Elle est indéniablement porteuse de positions à discuter, d'accords et de désaccords avec d'autres thèses présentes dans le champ des didactiques. En d'autres termes, elle tente de répondre, du mieux possible, à l'ouverture du débat souhaitée lors de la journée d'étude et prolongée par ce numéro de revue. Continuons le débat !

\section{NOTES}

1. Il s'agit de la journée d'étude "Didactique et/ou Didactiques. D'une question polémique à la construction d'un espace de problématisation » qui a eu lieu le 10 juin 2013 à l'IFE à Lyon. Cet article est la version remaniée de ma communication intitulée: «Didactiques et disciplines: les raisons d'un pluriel et les recherches en cours ».

2. L'alternance «je/nous» dans cet article renvoie à la difficulté de toujours bien démêler ce qui relève des avancées individuelles et des positions collectives du laboratoire Theodile. J'en profite pour remercier tous les membres de l'équipe pour les échanges qui m'ont permis de pousser toujours plus avant mes réflexions sur ces questions.

3. Parlant de manière de penser, de discourir et d'agir, je fais référence aux travaux importants de l'équipe de didactique du Français de Bordeaux, notamment à ceux de JeanPaul Bernié, Martine Jaubert, Maryse Rebière.

4. Voir, par exemple sur l'écriture, le travail de référence de Marie-Claude Penloup (1999).

5. Jean-Louis Martinand (2006, p. 362) avance d'ailleurs que "les didactiques ne sont pas des disciplines auto reproductrices $»$.

6. Sans oublier les références aux champs de pratiques sociales.

7. J'en ai effectué une présentation proche dans ma communication « La discipline en question(s) », effectuée le 21 mars 2013 à l'IFE à l'occasion du colloque en hommage à J.-P. Astolfi : Recherches et questions sur l'éducation aujourd'hui. J-.P. Astolfi, une référence d'actualité.

8. Mais pas uniquement à ceux-ci.

9. Conservatoires, chorales, clubs de sport, musées, bibliothèques...

10. Voir, par exemple, les discours médiatiques parfois apocalyptiques sur l'orthographe ou la lecture ou certains problèmes liés à l'enseignement de ce qui touche à la sexualité, à l'évolution des vivants ou encore à la Shoah.

11. Éducation Civique Juridique et Sociale, Travaux Personnels Encadrés, Itinéraires De Découverte...

12. Peut-être vu le poids des fonctionnements structurels de la forme scolaire (Vincent, 1980 et 2012; Vincent, dir. 1994).

13. Je n'ignore pas que la distinction proposée par Chervel peut être lue de différentes manières...

14. Voire « histoire-géographie-éducation civique».

15. Alternance terminologique qui n'est pas absente non plus des écrits de Chervel lui-même.

16. Textes officiels dont le statut légal peut d'ailleurs être fort variable. Voir, sur ce point, les travaux de référence de Roger-François Gauthier (2011).

17. Je reviendrai sur cette expression à propos des modes d'appropriation des disciplines.

18. Il reste cependant à déterminer quelles sont les tensions spécifiques et quelles sont les tensions partagées par les différentes disciplines scolaires.

19. La Sauvegarde du Nord est un des plus importants organismes de travail social de la Région Nord-Pas-de-Calais.

20.Cette notion est pourtant explicitement présente chez Chervel.

21. C'est-à-dire partageant des principes et des pratiques de fonctionnement avec les autres disciplines.

22. Par exemple, matières littéraires vs matières scientifiques ; EPS vs autres disciplines...

23. Au moins, celles que je perçois aujourd'hui.

24. Pour une première approche, voir Dabène et Rispail, 2008. Voir aussi Bishop (2013) sur la mise en perspective historique.

25. Voir, par exemple, Terrisse et Carnus, dir., 2009.

26. Voir, par exemple, Billières et Spanghero-Gaillard, 2005.

27. Voir, par exemple, Pastré 2008 ou Pastré, Mayen et Vergnaud, 2006

28. En effet, il ne me semble pas impossible de comprendre dans ce cadre le modèle d'émergence et de développement des didactiques en quatre époques, tel qu'il est construit par Lebeaume (2012), ou même le projet du Dictionnaire des concepts fondamentaux des didactiques. Mais la « didactique générale " (son existence, son statut, ses contenus, ses fonctions...) demeure pour moi une question ouverte. 


\section{RÉFÉRENCES}

Astolfi, J.-P. (1997). L'erreur, un outil pour enseigner. Paris : ESF.

Astolfi, J.-P. (2008). La saveur des savoirs. Disciplines et plaisir d'apprendre. Paris: ESF.

Billières, M., Spanghero-Gaillard, N. (2005). La didactique cognitive des langues : regards croisés des disciplines pour comprendre le « comment ». Parole, 34-36, 101137.

Bishop, M-F. (2013). Statut et fonctions de la mise en perspective historique dans la didactique du français. Note de synthèse pour l'Habilitation à Diriger des Recherches, université de Lille 3, Villeneuve d'Asq.

Chervel, A. (1988). L'histoire des disciplines scolaires. Réflexions sur un domaine de recherche. Histoire de l'éducation, 38, 59-119.

Chervel, A. (1998). La culture scolaire. Une approche historique. Paris: Belin.

Chervel, A. (2006). Histoire de l'enseignement du français du xviie au xxe siècle. Paris : Retz.

Chevallard, Y. (1985/1991). La transposition didactique. Du savoir savant au savoir enseigné. Grenoble : La Pensée Sauvage.

Cohen-Azria, C., Lahanier-Reuter, D., Reuter, Y. (dir.) (à paraitre). Conscience disciplinaire. Les représentations des disciplines à l'école primaire. Rennes: Presses Universitaires de Rennes.

Dabène, M., Rispail, M.-N. (2008). La sociodidactique : naissance et développement d'un courant au sein de la didactique du français en France. La Lettre de l'AIRDF, 42, 10-13.

Daunay, B., Reuter, Y. (2012). Penser et problématiser les contenus disciplinaires : un enjeu fondamental pour les didactiques. In B. Daunay, Y. Reuter, A. Thépaut, (dir.), Les contenus disciplinaires. Approches comparatistes, Villeneuve d'Ascq : Presses Universitaires du Septentrion (p. 21-34).

Gauthier, R.-F. (2011). "Malgoverno " éducatif et curriculaire en France. Fil rouge sur travaux (1985-2010). Note de synthèse pour l'Habilitation à diriger des recherches, université de Lyon 2, Lyon.

Lebeaume, J. (2012). La didactique de la technologie à la croisée des curriculums, des apprentissages et des enseignements disciplinaires, dans une perspective socio-historique générale. In M.-L. Elalouf, A. Robert, M.-F. Bishop (dir). Les didactiques en question(s), État des lieux et perspectives pour la recherche et la formation (p. 48-55). Bruxelles: De Boeck.

Lesné, M. (1977). Travail pédagogique et formation d'adultes. Paris : PUF.

Martinand, J.-L. (1987). Quelques remarques sur les didactiques des disciplines. Les Sciences de l'éducation Pour l'ère nouvelle, vol. 1-2, 23-35.

Martinand, J.-L. (2006). Didactique et didactiques. Esquisse problématique. In J. Beillerot \& N. Mosconi, (dir.). Traité des sciences et des pratiques de l'éducation (p. 353-367). Paris : Dunod.
Mercier, A., Sensevy, G., Schubauer-Leoni, M.-L. (2002). Vers une didactique comparée. Revue Française de Pédagogie, 141, 5-16.

Pastré Pierre (2008) : La didactique professionnelle : origines, fondements, perspectives, Travail et apprentissages, $\mathrm{n}^{\circ} 1$, Dijon, Éditions Raisons et Passions, 9-21.

Pastré, P., Mayen, P., Vergnaud, G. (2006). La didactique professionnelle. Note de synthèse. Revue Française de Pédagogie, 136, 145-198.

Penloup, M.-C. (1999). Lécriture extrascolaire des collégiens. Des constats aux perspectives didactiques. Paris : ESF.

Reuter, Y. (2003). La représentation de la discipline ou la conscience disciplinaire. La Lettre de la DFLM, 32,1822.

Reuter, Y. (2005a). Analyser le faire des élèves dans une perspective didactique. Les Cahiers Theodile, 6, 33-40.

Reuter, Y. (2005b). Les enjeux du français : questions pour la didactique. Recherches, 43, 25-38.

Reuter, Y. (2007). La conscience disciplinaire. Présentation d'un concept. Éducation et didactique, vol. 1, 2, 57-71.

Reuter, Y. (2008). Interroger l'appareillage conceptuel de la didactique du français. La Lettre de l'AIRDF, 42, 18-21.

Reuter, Y. (2010). Définition et transmission des savoirs scolaires : statut des tensions dans une approche didactique. In R. Malet (Éd.). École, médiations et réformes curriculaires (p. 39-46). Bruxelles : De Boeck.

Reuter, Y. (2011). Penser la perspective didactique : la question de l'articulation entre disciplinaire, pédagogique et scolaire. In B. Daunay, Y. Reuter, B. Schneuwly (dir.). Les concepts et les méthodes en didactique du français (p. 35-60). Namur : Presses universitaires de Namur.

Reuter, Y. (2013). Panser l'erreur à l'école. De l'erreur au dysfonctionnement. Villeneuve d'Ascq : Presses Universitaires du Septentrion.

Reuter, Y. (dir.) (2007/2010). Dictionnaire des concepts fondamentaux des didactiques. Bruxelles: De Boeck (troisième édition, 2013).

Reuter, Y., Daunay, B., Delcambre, I., Cohen-Azria, C., Lahanier-Reuter, D. (2011). Contribution à un dialogue. Réponse des auteurs de l'ouvrage recensé. Éducation et Didactique, vol. 5, 1, 121-125.

Reuter, Y., Delcambre, I. (2006). Lobjet enseigné comme objet à construire. Une discussion critique. In dans B. Schneuwly, T. Thévenaz-Christen (dir.). Analyse des objets enseignés. Le cas du français (p. 233-252). Bruxelles : De Boeck.

Reuter, Y., Lahanier-Reuter, D. (2004/2007). Lanalyse de la discipline : quelques problèmes pour la recherche en didactique, Communication au $9^{e}$ colloque de l'AIRDF, Québec, août 2004, reprise dans E. Falardeau, C. Fisher, C. Simard, N. Sorin (dir.). La didactique du français. Les voies actuelles de la recherche (p. 27-42). Québec: Presses de l'université Laval.

Terrisse, A., Carnus, M.-F. (dir.) (2009). Didactique clinique de l'éducation physique et sportive (EPS). Quels enjeux de savoirs? Bruxelles: De Boeck. 
Thevenaz-Christen, T. (2008). Au cœur de la forme scolaire, la discipline. L'exemple du français parlé. Revue suisse des Sciences de l'éducation, 2, 299-324.

Verret, M. (1975). Le temps des études. Paris : Honoré Champion.

Vincent, G. (1980). L'école primaire française. Lyon : Presses Universitaires de Lyon.

Vincent, G. (2012). La forme scolaire : débats et mises au point ( $1^{\text {re }}$ partie). Entretien avec B. Courtebras et Y. Reuter. Recherches en didactiques, 13, 109-135.

Vincent, G. (dir.) (1994). L'éducation prisonnière de la forme scolaire? Scolarisation et socialisation dans les sociétés industrielles. Lyon : Presses universitaires de Lyon. 